\title{
Anatomic study of verumontanum during endoscopic surgeries in patients with benign prostatic hyperplasia
}

Henrique Barbosa de Menezes ${ }^{1,2}$, Francisco José Barcellos Sampaio ${ }^{1}$, José Anacleto Dutra de Resende Júnior ${ }^{2}$, Rodrigo Ribeiro Vieiralves ${ }^{1}$, Fernando Salles da Silva Filho ${ }^{2}$, Edilaine Alves ${ }^{1}$, Luciano Alves Favorito $^{1}$

${ }^{1}$ Unidade de Pesquisa Urogenital, Universidade Estadual do Rio de Janeiro - UERJ, Rio de Janeiro, RJ, Brasil; ${ }^{2}$ Setor de Urologia, Hospital Federal de Lagoa, Rio de Janeiro, RJ, Brasil

\section{ABSTRACT}

Introduction and objective: To evaluate changes in verumontanum anatomy in patients with benign prostatic hyperplasia (BPH) who used 5-alpha reductase inhibitors (5-ARIs) and to propose an anatomical classification of the verumontanum.

Materials and Methods: We studied 86 patients with BPH and 7 patients without the disease (age under 40 years-old who underwent kidney or ureteral lithotripsy). Of the patients with BPH, 34 (mean age=67.26) had 5-ARIs use and 52 (mean age=62.69) did not use the drug. During surgeries, photographs of the seminal colliculus were taken and later, with the aid of software (Image J), the length (longitudinal diameter) and width (transverse diameter) of the verumontanum were measured in all patients. During the procedure, we evaluated the different types of verumontanum. For statistical analysis, the R-Project software was used. Results: In the group of patients with BPH who were taking medication (group 1), the mean measures of length and width of the verumontanum were $4.69 \mathrm{~mm}$ and $2.94 \mathrm{~mm}$ respectively. In the group of patients with BPH who did not use the drug (group 2), the mean diameters were $4.54 \mathrm{~mm}$ and $3.20 \mathrm{~mm}$ respectively. In the control group (group 3), the average length and width were $5.63 \mathrm{~mm}$ and $4.11 \mathrm{~mm}$ respectively. There was an increase in longitudinal and transverse measurements of the control group with an increase in body mass index (BMI) ( $p=0.0001$ and $p=0.035$ respectively). In addition, there was a reduction in transverse diameter in the group of BPH using 5-ARI with increased prostate volume $(p=0.010)$. We found five different verumontanum types: "volcano" (51.61\%), "lighthouse" (24.73\%), "whale tail" (12.90\%), "hood" (5.38\%) and "castle door" (5.38\%), which we propose as an anatomical classification.

Conclusion: Veromontanum has smaller measurements in patients with BPH regardless of treatment. In the control group, there was an increase in verumontanum diameters with an increase in BMI. The volcano type of verumontanum was the most frequent regardless of groups and BMI.

\section{ARTICLE INFO}

José Anacleto Dutra Júnior

https://orcid.org/0000-0001-7396-4351

Keywords:

Prostatic Hyperplasia; Lower Urinary Tract Symptoms;

5-alpha Reductase Inhibitors

Int Braz J Urol. 2020; 47: 308-21

Submitted for publication:

January 20, 2020

Accepted after revision:

March 09, 2020

Published as Ahead of Print: October 10, 2020

\section{INTRODUCTION}

The verumontanum (seminal colliculus) is a bulge distal to the urethral crest that presents the prostatic utricle (remnant of the Muller ducts) and the two ejaculatory ducts (1). The verumontanum originates from the endoderm of the bladder part of the urogenital sinus and has great anatomical and func- 
tional importance due to the presence of ejaculatory ducts, fundamental structures for semen elimination. Thus, this structure plays an important role in reproduction (2). It can be affected by problems such as cysts or polyps, which lead to symptoms of emptying, dysuria, hematuria, infertility, hemospermia, prostatitis and urinary tract infection (3).

Although there are anatomical classifications of the prostate (McNeal and Randall) $(4,5)$ and classification of the prostate utricle (6), so far no classification of the seminal colliculus has been created.

Benign prostatic hyperplasia (BPH) is one of the most common diseases in men, with progressive incidence according to age. BPH leads to lower urinary tract symptoms (LUTS) due to intra-bladder obstruction (7). Among the medications used to treat $\mathrm{BPH}, 5$-alpha-reductase inhibitors (5-ARIs) are prominent because they are able to alter the natural history of the disease by decreasing prostate volume (8). There are two FDA-authorized 5-ARIs drugs: finasteride and dutasteride. Finasteride acts by inhibiting type 2 enzyme, while dutasteride inhibits types 1 and 2. However, this class of drugs has side effects such as ejaculatory disorders and reduced semen volume (8-10).

Previous studies analyzing the anatomy of the verumontanum in $\mathrm{BPH}$ and in patients with normal prostates are scarce in the literature. We hypothesized that the verumontanum anatomy could be altered in patients with $\mathrm{BPH}$ due to the use of 5-alpha-reductase inhibitors, which could justify side effects such as ejaculatory disorders. Although, that is not the purpose of our work.

The aim of this paper is to create an anatomical endoscopic classification for verumontanum and to assess changes in verumontanum anatomy in patients with benign prostatic hyperplasia (BPH) using 5-alpha-reductase inhibitors, such as: assess whether the 5-ARIs alter the size or anatomy of the verumontanum, assess whether BPH increase the size or change the anatomy of the verumontanum, compare the size of the verumontanum with age, the body mass index (BMI) and the prostate weight.

\section{MATERIALS AND METHODS}

The experimental protocol described here was approved by the committee for ethical human experimentation of our university. This study was carried out in accordance with the ethical standards of the hospital's institutional committee on human experimentation (opinion number 3.233.220).

This is an anatomical, observational, analytical, prospective and non-randomized study, carried out at the Federal Hospital of Lagoa, started in March 2018 and completed in October 2019. We studied 86 patients with $\mathrm{BPH}$ (age 41 to 85 years, mean $=64.5$ years) and 7 patients without $\mathrm{BPH}$, who formed the control group (age 29 to 38 years, mean $=32.71$ years). Of the patients with BPH, 34 used 5-ARIs (16 used finasteride and 18 used dutasteride, wich composed the Group 1) and 52 did not use this class of drugs (Group 2). The average age of group 1 was 67 years and the average age of group 2 was 62 years. All the patients in the study were evaluated by the same professional, who applied the same questionnaire. Data were collected such as age, height, weight, body mass index, prostate weight, alpha-blocker use, 5-alpha-reductase inhibitor use, presence of systemic arterial hypertension and diabetes mellitus, and delayed bladder catheter (DBC) use.

Inclusion criteria: Patients with $\mathrm{BPH}$ who underwent transurethral resection (TUR) of the prostate or bladder and patients younger than 40 years without BPH who underwent an endoscopic procedure to treat urolithiasis composed the control group (because the literature shows that at this age occurs a significant increase in the prevalence of $\mathrm{BPH}$, as well as in lower urinary tract symptoms associated with $\mathrm{BPH}$ ) (7).

Exclusion criteria: All patients with any other prostate pathology (prostate cancer, prostatitis, prostatic cyst, etc.), patients with BPH who used finasteride or dutasteride for less than 6 months (because the literature shows that at this moment the drugs start to have the best effect) (11), as well as patients undergoing any minimally invasive surgical treatment of the prostate. In our study, all patients who used 5-ARIs were also using alpha-blockers.

In order to standardize the performance of TURs so that the technique used in the introduction of the resectoscope was always the same, all patients were operated by the same surgeon. The resectoscope used was Olympus ${ }^{\circledR} 26$ French (Fr) - continuous flow. The electrodes used were "loop" type. The generator used was the Olympus ${ }^{\circledR}$ bipolar plasma. During transurethral resection surgery, photographs of the veru- 
montanum were taken $(40 \mathrm{~cm}$ distance between the camera and the monitor screen) and the images were analyzed using Image J version $1.46 \mathrm{r}$, with its plug-in (http://rsb.info.nih.gov/ij/). The longitudinal and transverse diameters of the seminal colliculus were measured using the distance between the two ends of the resectoscopic loop, which was determined prior to surgery individually as a measurement parameter. The distance of the resection loop and the optics was standardized $(1 \mathrm{~cm})$. In the case of patients in the control group, the measurement was made using the diameter of a ureteral catheter (previously known measure) as a parameter for verumontanum diameter measurement (Figure-1). All verumontanums were initially photographed without the resection loop and without the ureteral catheter in the visual field so that they could be evaluated to standardize a classification of their anatomy.

After completing the verumontanum measurements, comparisons were made between the following groups: patients with $\mathrm{BPH}$ who used 5-alpha-reductase inhibitors (group 1), patients with BPH

who did not use 5-alpha-reductase inhibitors (group 2), and patients under 40 years of age, without BPH, undergoing the endoscopic procedure to treat urolithiasis (group 3, control). In addition, comparisons of verumontanum size with age, BMI and prostate weight were performed. After the analysis of all verumontanums, we proposed a new classification for the organ's morphology.

Statistical analysis was performed using the R-Project software, version 3.5.3. The Kruskal-Wallis test and Dunn's post-test were used to verify if there was a statistically significant difference between the means of the variables. The Mann-Whitney mean comparison test was used to evaluate the prostate size variables present in groups 1 and 2. Simple linear correlations were calculated to compare verumontanum measurements with variables in the three groups. We considered $p$-values $<0.05$ as statistically significant.

\section{RESULTS}

Figure 1 - The figure shows the measurement of verumontanum diameters of groups 1, 2 and 3. A) Standardization of recurrent distance in groups 1 and 2 (distance between the two ends of the resection loop). B) Measurement of the longitudinal diameter of the verumontanum of groups 1 and 2. C) Standardization of the recognized distance of group 3 (ureteral catheter diameter). D) Verification of the longitudinal diameter of the verumontanum.
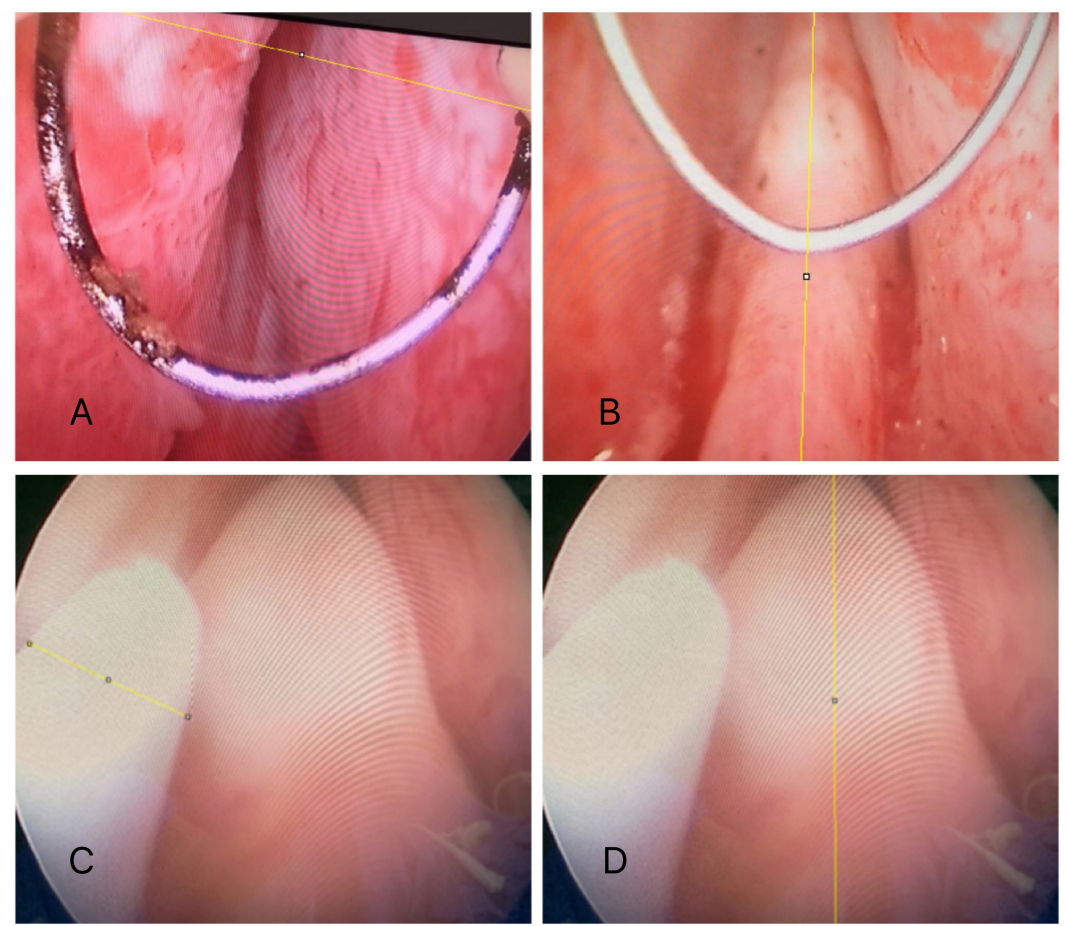
Table 1 - All data and variables of all patients.

\begin{tabular}{|c|c|c|c|c|c|c|c|c|c|c|}
\hline \multicolumn{11}{|c|}{ Data and variables of patients with BPH } \\
\hline Pt. & Age & LDV & TDV & $\begin{array}{c}\text { Verum. } \\
\text { Type }\end{array}$ & Prostate Weight & 5 ARI & $\begin{array}{c}\text { OBES./BMI } \\
\left(\mathrm{kg} / \mathrm{m}^{2}\right)\end{array}$ & SAH & DM & DBC \\
\hline 1 & 55 & $2.75 \mathrm{~mm}$ & $2.20 \mathrm{~mm}$ & WT & $60 \mathrm{~g}$ & Yes & Yes/31.37 & No & No & No \\
\hline 2 & 66 & $3.19 \mathrm{~mm}$ & $3.3 \mathrm{~mm}$ & V & $75 \mathrm{~g}$ & Yes & No/24.91 & No & No & Yes \\
\hline 3 & 52 & $2.81 \mathrm{~mm}$ & $3.39 \mathrm{~mm}$ & WT & $54 \mathrm{~g}$ & Yes & No/27.3 & Yes & Yes & No \\
\hline 4 & 72 & $3.71 \mathrm{~mm}$ & $3.5 \mathrm{~mm}$ & $C D$ & $55 \mathrm{~g}$ & Yes & $\mathrm{No} / 24.0$ & No & No & Yes \\
\hline 5 & 68 & $7.11 \mathrm{~mm}$ & $2.2 \mathrm{~mm}$ & $\mathrm{H}$ & $46 \mathrm{~g}$ & Yes & Yes/38.4 & Yes & Yes & No \\
\hline 6 & 64 & $6.6 \mathrm{~mm}$ & $3.12 \mathrm{~mm}$ & WT & $47 \mathrm{~g}$ & Yes & No/23.8 & Yes & Yes & Yes \\
\hline 7 & 66 & $2.91 \mathrm{~mm}$ & $2.26 \mathrm{~mm}$ & V & $54 \mathrm{~g}$ & Yes & No/29.72 & No & No & Yes \\
\hline 8 & 68 & $5.18 \mathrm{~mm}$ & $3.23 \mathrm{~mm}$ & $\mathrm{H}$ & $63 \mathrm{~g}$ & Yes & Yes/34.15 & Yes & No & Yes \\
\hline 9 & 63 & $2.88 \mathrm{~mm}$ & $2.04 \mathrm{~mm}$ & $C D$ & $65 \mathrm{~g}$ & Yes & No/22.59 & Yes & Yes & No \\
\hline 10 & 58 & $4.56 \mathrm{~mm}$ & $3.88 \mathrm{~mm}$ & $C D$ & $40 \mathrm{~g}$ & Yes & No/26.81 & Yes & No & No \\
\hline 11 & 79 & $6.31 \mathrm{~mm}$ & $4.2 \mathrm{~mm}$ & V & $57 \mathrm{~g}$ & Yes & No/29.29 & Yes & No & No \\
\hline 12 & 68 & $3.92 \mathrm{~mm}$ & $4.0 \mathrm{~mm}$ & V & $50 \mathrm{~g}$ & No & No/22.94 & No & No & No \\
\hline 13 & 77 & $4.92 \mathrm{~mm}$ & $3.0 \mathrm{~mm}$ & V & $140 \mathrm{~g}$ & No & $\mathrm{No} / 26.9$ & Yes & No & No \\
\hline 14 & 72 & $8.31 \mathrm{~mm}$ & $2.51 \mathrm{~mm}$ & L & $40 \mathrm{~g}$ & No & No/20.06 & No & No & No \\
\hline 15 & 66 & $4.37 \mathrm{~mm}$ & $4.40 \mathrm{~mm}$ & WT & $32 g$ & Yes & No/23.99 & No & No & No \\
\hline 16 & 68 & $6.91 \mathrm{~mm}$ & $3.24 \mathrm{~mm}$ & $\mathrm{H}$ & $63 \mathrm{~g}$ & Yes & No/25.0 & Yes & No & Yes \\
\hline 17 & 56 & $6.17 \mathrm{~mm}$ & $3.95 \mathrm{~mm}$ & L & $65 \mathrm{~g}$ & Yes & No/29.4 & Yes & No & No \\
\hline 18 & 78 & $5.36 \mathrm{~mm}$ & $2.11 \mathrm{~mm}$ & WT & $66 \mathrm{~g}$ & Yes & No/23.6 & Yes & No & Yes \\
\hline 19 & 63 & $4.51 \mathrm{~mm}$ & $2.90 \mathrm{~mm}$ & V & $40 \mathrm{~g}$ & No & $\mathrm{No} / 25.0$ & No & No & Yes \\
\hline 20 & 68 & $5.29 \mathrm{~mm}$ & $2.43 \mathrm{~mm}$ & V & $51 \mathrm{~g}$ & No & $\mathrm{No} / 25.9$ & No & No & Yes \\
\hline 21 & 60 & $5.33 \mathrm{~mm}$ & $3.75 \mathrm{~mm}$ & V & $41 \mathrm{~g}$ & No & $\mathrm{No} / 22.7$ & No & No & Yes \\
\hline 22 & 52 & $7.28 \mathrm{~mm}$ & $2.32 \mathrm{~mm}$ & $\mathrm{~L}$ & $70 \mathrm{~g}$ & Yes & $\mathrm{No} / 25.0$ & Yes & No & Yes \\
\hline 23 & 68 & $7.29 \mathrm{~mm}$ & $3.15 \mathrm{~mm}$ & WT & $53 g$ & Yes & $\mathrm{No} / 17.5$ & Yes & No & Yes \\
\hline
\end{tabular}




\begin{tabular}{|c|c|c|c|c|c|c|c|c|c|c|}
\hline 24 & 69 & $3.79 \mathrm{~mm}$ & $2.75 \mathrm{~mm}$ & V & $40 \mathrm{~g}$ & No & Yes/34.11 & Yes & Yes & No \\
\hline 25 & 68 & $4.70 \mathrm{~mm}$ & $4.17 \mathrm{~mm}$ & V & $66 \mathrm{~g}$ & No & $\mathrm{No} / 24.9$ & No & No & Yes \\
\hline 26 & 60 & $3.31 \mathrm{~mm}$ & $3.07 \mathrm{~mm}$ & V & $31 \mathrm{~g}$ & No & $\mathrm{No} / 28.5$ & Yes & No & No \\
\hline 27 & 67 & $4.5 \mathrm{~mm}$ & $3.06 \mathrm{~mm}$ & V & $69 g$ & No & No/23.14 & No & No & Yes \\
\hline 28 & 62 & $4.15 \mathrm{~mm}$ & $3.81 \mathrm{~mm}$ & V & $67 g$ & Yes & No/22.9 & Yes & Yes & No \\
\hline 29 & 78 & $3.57 \mathrm{~mm}$ & $1.24 \mathrm{~mm}$ & $\mathrm{H}$ & $100 \mathrm{~g}$ & Yes & No/25.8 & Yes & No & No \\
\hline 30 & 62 & $2.17 \mathrm{~mm}$ & $2.4 \mathrm{~mm}$ & V & $84 \mathrm{~g}$ & Yes & No/19.0 & Yes & Yes & No \\
\hline 31 & 65 & $4.03 \mathrm{~mm}$ & $1.7 \mathrm{~mm}$ & $L$ & $67 g$ & Yes & No/24.9 & No & No & Yes \\
\hline 32 & 64 & $6.31 \mathrm{~mm}$ & $4.14 \mathrm{~mm}$ & $L$ & $20 \mathrm{~g}$ & No & No/27.0 & No & No & No \\
\hline 33 & 72 & $5.87 \mathrm{~mm}$ & $3.14 \mathrm{~mm}$ & $L$ & $52 g$ & No & No/28.6 & No & Yes & No \\
\hline 34 & 67 & $2.24 \mathrm{~mm}$ & $2.35 \mathrm{~mm}$ & V & $33 g$ & No & Yes/31.0 & No & No & No \\
\hline 35 & 72 & $5.91 \mathrm{~mm}$ & $4.97 \mathrm{~mm}$ & $C D$ & $44 g$ & No & Yes/45.6 & Yes & No & No \\
\hline 36 & 74 & $2.6 \mathrm{~mm}$ & $2.4 \mathrm{~mm}$ & WT & $30 \mathrm{~g}$ & No & No/23.0 & Yes & Yes & No \\
\hline 37 & 81 & $3.19 \mathrm{~mm}$ & $2.46 \mathrm{~mm}$ & WT & $86 \mathrm{~g}$ & Yes & No/23.0 & Yes & No & No \\
\hline 38 & 71 & $4.59 \mathrm{~mm}$ & $1.96 \mathrm{~m}$ & $L$ & $59 g$ & Yes & Yes/32.0 & Yes & No & No \\
\hline 39 & 74 & $5.59 \mathrm{~mm}$ & $2.62 \mathrm{~mm}$ & $L$ & $48 \mathrm{~g}$ & No & No/28.0 & Yes & No & No \\
\hline 40 & 59 & $4.77 \mathrm{~m}$ & $3.19 \mathrm{~mm}$ & V & $47 \mathrm{~g}$ & Yes & No/22.8 & No & No & No \\
\hline 41 & 70 & $10 \mathrm{~mm}$ & $6.61 \mathrm{~mm}$ & V & $33 g$ & No & No/24.4 & Yes & No & No \\
\hline 42 & 68 & $4.74 \mathrm{~mm}$ & $3.75 \mathrm{~mm}$ & V & $41 \mathrm{~g}$ & Yes & No/26.0 & No & No & No \\
\hline 43 & 71 & $4.81 \mathrm{~mm}$ & $2.99 \mathrm{~mm}$ & V & $37 \mathrm{~g}$ & Yes & No/26.0 & No & No & Yes \\
\hline 44 & 79 & $5.34 \mathrm{~mm}$ & $2.77 \mathrm{~mm}$ & $\mathrm{~L}$ & $55 \mathrm{~g}$ & Yes & No/24.0 & No & Yes & No \\
\hline 45 & 60 & $5.65 \mathrm{~mm}$ & $3.92 \mathrm{~mm}$ & V & $20 \mathrm{~g}$ & Yes & Yes/34.6 & Yes & No & No \\
\hline 46 & 70 & $2.57 \mathrm{~mm}$ & $2.51 \mathrm{~mm}$ & WT & $42 g$ & Yes & No/21.5 & Yes & No & Yes \\
\hline 47 & 58 & $7.93 \mathrm{~mm}$ & $6.82 \mathrm{~mm}$ & V & $20 \mathrm{~g}$ & No & $\mathrm{No} / 24.9$ & Yes & No & No \\
\hline 48 & 82 & $4.93 \mathrm{~mm}$ & $3.25 \mathrm{~mm}$ & $L$ & $40 \mathrm{~g}$ & No & No/24.9 & Yes & No & No \\
\hline 49 & 60 & $4.49 \mathrm{~mm}$ & $3.94 \mathrm{~mm}$ & V & $63 \mathrm{~g}$ & No & Yes/31.4 & Yes & Yes & No \\
\hline
\end{tabular}




\begin{tabular}{|c|c|c|c|c|c|c|c|c|c|}
\hline 50 & 77 & $3.59 \mathrm{~mm}$ & $2.16 \mathrm{~mm}$ & V & $36 \mathrm{~g}$ & Yes & No/28.0 & Yes & Yes \\
\hline 51 & 44 & $3.1 \mathrm{~mm}$ & $2.85 \mathrm{~mm}$ & $C D$ & $25 \mathrm{~g}$ & No & No/25.53 & No & No \\
\hline 52 & 69 & $4.47 \mathrm{~mm}$ & $2.48 \mathrm{~mm}$ & $\mathrm{~L}$ & $56 \mathrm{~g}$ & Yes & No/27.68 & Yes & No \\
\hline 53 & 70 & $4.92 \mathrm{~mm}$ & $2.30 \mathrm{~mm}$ & $\mathrm{~L}$ & $25 \mathrm{~g}$ & No & No/27.15 & Yes & No \\
\hline 54 & 80 & $3.85 \mathrm{~mm}$ & $2.13 \mathrm{~mm}$ & V & $158 \mathrm{~g}$ & Yes & No/22.03 & Yes & No \\
\hline 55 & 48 & $3.06 \mathrm{~mm}$ & $2.67 \mathrm{~mm}$ & V & $27 \mathrm{~g}$ & No & No/28.3 & Yes & No \\
\hline 56 & 74 & $4.32 \mathrm{~mm}$ & $3.29 \mathrm{~mm}$ & V & $24 g$ & No & No/26.98 & Yes & No \\
\hline 57 & 57 & $4.58 \mathrm{~mm}$ & $3.15 \mathrm{~mm}$ & V & $35 \mathrm{~g}$ & No & No/26.49 & No & No \\
\hline 58 & 56 & $4.42 \mathrm{~mm}$ & $1.59 \mathrm{~mm}$ & L & $35 g$ & No & Yes/32.11 & Yes & No \\
\hline 59 & 70 & $4.22 \mathrm{~mm}$ & $2.37 \mathrm{~mm}$ & L & $51 \mathrm{~g}$ & No & Yes/32.0 & Yes & No \\
\hline 60 & 46 & $2.22 \mathrm{~mm}$ & $2.35 \mathrm{~mm}$ & V & $29 g$ & No & No/23.87 & Yes & No \\
\hline 61 & 49 & $4.92 \mathrm{~mm}$ & $4.53 \mathrm{~mm}$ & V & $27 g$ & No & No/27.76 & No & No \\
\hline 62 & 41 & $4.49 \mathrm{~mm}$ & $3.23 \mathrm{~mm}$ & V & $30 \mathrm{~g}$ & No & No/20.76 & No & No \\
\hline 63 & 58 & $2.45 \mathrm{~mm}$ & $2.66 \mathrm{~mm}$ & V & $19 g$ & No & No/26.34 & No & No \\
\hline 64 & 56 & $2.36 \mathrm{~mm}$ & $5.09 \mathrm{~mm}$ & V & $35 g$ & No & No/22.34 & No & No \\
\hline 65 & 83 & $4.25 \mathrm{~mm}$ & $3.13 \mathrm{~mm}$ & V & $75 \mathrm{~g}$ & No & No/23.62 & No & No \\
\hline 66 & 76 & $8.0 \mathrm{~mm}$ & $1.76 \mathrm{~mm}$ & $\mathrm{H}$ & $30 \mathrm{~g}$ & No & No/24.7 & Yes & No \\
\hline 67 & 61 & $3.80 \mathrm{~mm}$ & $1.48 \mathrm{~mm}$ & $\mathrm{~L}$ & $30 \mathrm{~g}$ & No & No/22.34 & No & No \\
\hline 68 & 63 & $5.34 \mathrm{~mm}$ & $4.21 \mathrm{~mm}$ & V & $31 \mathrm{~g}$ & No & No/27.71 & No & No \\
\hline 69 & 50 & $4.94 \mathrm{~mm}$ & $3.36 \mathrm{~mm}$ & V & $28 \mathrm{~g}$ & No & No/29.58 & Yes & Yes \\
\hline 70 & 42 & $3.17 \mathrm{~mm}$ & $3.84 \mathrm{~mm}$ & V & $30 \mathrm{~g}$ & No & Yes/34.33 & Yes & Yes \\
\hline 71 & 52 & $3.65 \mathrm{~mm}$ & $2.56 \mathrm{~mm}$ & V & $50 \mathrm{~g}$ & No & No/25.30 & Yes & No \\
\hline 72 & 68 & $3.03 \mathrm{~mm}$ & $3.06 \mathrm{~mm}$ & v & $65 \mathrm{~g}$ & No & Yes/31.37 & Yes & No \\
\hline 73 & 72 & $2.22 \mathrm{~mm}$ & $1.74 \mathrm{~mm}$ & V & $42 g$ & No & No/27.16 & Yes & Yes \\
\hline 74 & 49 & $4.03 \mathrm{~mm}$ & $2.54 \mathrm{~mm}$ & L & $38 \mathrm{~g}$ & No & No/29.41 & No & No \\
\hline
\end{tabular}




\begin{tabular}{ccccccccccc}
75 & 47 & $4.37 \mathrm{~mm}$ & $4.42 \mathrm{~mm}$ & WT & $39 \mathrm{~g}$ & No & No/28.32 & Yes & No & No \\
76 & 77 & $2.68 \mathrm{~mm}$ & $2.64 \mathrm{~mm}$ & $\mathrm{~V}$ & $24 \mathrm{~g}$ & No & No/22.72 & No & No & No \\
77 & 85 & $4.45 \mathrm{~mm}$ & $4.33 \mathrm{~mm}$ & $\mathrm{~V}$ & $40 \mathrm{~g}$ & Yes & No/26.36 & No & No & No \\
78 & 44 & $4.28 \mathrm{~mm}$ & $2.25 \mathrm{~mm}$ & $\mathrm{~L}$ & $27 \mathrm{~g}$ & No & No/27.68 & No & No & No \\
79 & 61 & $2.42 \mathrm{~mm}$ & $2.08 \mathrm{~mm}$ & $\mathrm{~V}$ & $75 \mathrm{~g}$ & No & No/24.77 & Yes & Yes & No \\
80 & 51 & $8.08 \mathrm{~mm}$ & $3.73 \mathrm{~mm}$ & $\mathrm{~L}$ & $75 \mathrm{~g}$ & Yes & No/24.38 & No & No & No \\
81 & 76 & $3.75 \mathrm{~mm}$ & $2.65 \mathrm{~mm}$ & $\mathrm{~V}$ & $54 \mathrm{~g}$ & No & Yes/31.57 & Yes & Yes & No \\
82 & 68 & $4.38 \mathrm{~mm}$ & $3.31 \mathrm{~mm}$ & $\mathrm{WT}$ & $38 \mathrm{~g}$ & No & No/22.86 & No & No & No \\
83 & 53 & $11.26 \mathrm{~mm}$ & $5.6 \mathrm{~mm}$ & $\mathrm{~L}$ & $30 \mathrm{~g}$ & No & No/29.2 & No & No & No \\
84 & 46 & $3.87 \mathrm{~mm}$ & $2.87 \mathrm{~mm}$ & $\mathrm{~L}$ & $30 \mathrm{~g}$ & No & No/25.0 & Yes & No & No \\
85 & 66 & $2.85 \mathrm{~mm}$ & $1.64 \mathrm{~mm}$ & $\mathrm{~L}$ & $35 \mathrm{~g}$ & No & No/29.32 & Yes & Yes & No \\
86 & 72 & $4.41 \mathrm{~mm}$ & $3.46 \mathrm{~mm}$ & $\mathrm{~V}$ & $40 \mathrm{~g}$ & No & No/24.0 & Yes & Yes & No \\
\hline
\end{tabular}

\section{Data and variables of patients without BPH (control group)}

\begin{tabular}{ccccccccc}
\hline Pt. & Age & LDV & TDV & $\begin{array}{c}\text { Verum. } \\
\text { Type }\end{array}$ & $\begin{array}{c}\text { OBES./BMI } \\
\left(\mathrm{kg} / \mathrm{m}^{2}\right)\end{array}$ & SAH & DM & DBC \\
1 & 29 & $16.6 \mathrm{~mm}$ & $10 \mathrm{~mm}$ & $\mathrm{~L}$ & Yes $/ 34.0$ & No & No & No \\
2 & 37 & $3.14 \mathrm{~mm}$ & $2.0 \mathrm{~mm}$ & $\mathrm{~V}$ & $\mathrm{No} / 25.9$ & No & No & No \\
3 & 38 & $2.77 \mathrm{~mm}$ & $2.12 \mathrm{~mm}$ & $\mathrm{~V}$ & No/ 25.39 & No & No & No \\
4 & 34 & $2.79 \mathrm{~mm}$ & $1.74 \mathrm{~mm}$ & $\mathrm{~V}$ & No $/ 25.9$ & No & No & No \\
5 & 29 & $4.58 \mathrm{~mm}$ & $2.81 \mathrm{~mm}$ & WT & No $/ 25.6$ & No & No & No \\
6 & 30 & $5.28 \mathrm{~mm}$ & $7.65 \mathrm{~mm}$ & $\mathrm{~V}$ & No $/ 26.06$ & No & No & No \\
7 & 32 & $4.26 \mathrm{~mm}$ & $2.46 \mathrm{~mm}$ & $\mathrm{~L}$ & No/26.77 & No & No & No \\
\hline
\end{tabular}

$\mathbf{P t}=$ Patient; $\mathbf{L D V}=$ Longitudinal diameter of the verumontanum; $\mathbf{T D V}=$ transverse diameter of the verumontanum; $5 \mathrm{ARI}=5$-alpha-reductase inhibitor; $0 \mathrm{BES}$. $/ \mathrm{BMI}=0 \mathrm{besity} /$ Body Mass Index; $\mathbf{S A H}=$ systemic arterial hypertension; DM = Diabetes Mellitus; $\mathbf{D B C}=$ delayed bladder catheter; $\mathbf{m m}=$ millimeter; $\mathbf{g}=$ gram; $\mathbf{V}=$ volcano; $\mathbf{W T}=$ Whale Tail; $\mathbf{L}=$ Lighthouse; $\mathbf{H}=$ Hood; $\mathbf{C D}=$ Castle Door.

Note: Prostate volume was not included in the control group because in this age group there is no routine investigation of benign prostatic hyperplasia. 
All variables studied are presented in $\mathrm{Ta}-$ ble-1. The mean, standard deviation and median of BMI, prostate weight and verumontanum measurements are shown in Table- 2 .

In the control group, there was an increase in longitudinal $\left(\mathrm{R}^{2}=0.9791 ; \mathrm{p}=0.0013\right)$ and transverse $\left(R^{2}=0.9777 ; p=0.0014\right)$ measurements of the verumontanum with rising body mass index (BMI), with statistical significance. However, in the comparison according to age, verumontanum diameters decreased (longitudinal diameter $\left(\mathrm{R}^{2}=0.3403 ; \mathrm{p}=0.1692\right)$ and transverse diameter $\left(R^{2}=0.4221 ; p=0.1142\right)$ as age increased (Figure-2).

In BPH patients who used 5-alpha-reductase inhibitors (group 1), when comparison of the verumontanum measurements and BMI revealed na increase in longitudinal diameter $\left(\mathrm{R}^{2}=0.0285\right.$; $\mathrm{p}=0.3397$ ) and a slight increase in transverse diameter $\left(R^{2}=0.0005 ; p=0.8986\right)$ with an increase in BMI. In this group there was a reduction in longitudinal $\left(\mathrm{R}^{2}=0.0238 ; \mathrm{p}=0.3833\right)$ and transverse $\left(\mathrm{R}^{2}=0.0325 ; \mathrm{p}=0.3080\right)$ diameters with increasing age. In the comparson with prostate weight, there was a reduction in longitudinal $\left(R^{2}=0.0237\right.$; $\mathrm{p}=0.3852)$ and transverse $\left(\mathrm{R}^{2}=0.1864 ; \mathrm{p}=0.0108\right)$ diameters as the prostate weight increased, but only the transverse diameter was statistically significant (Figure-3).

In the control group, there was an increase in longitudinal $\left(\mathrm{R}^{2}=0.9791 ; \mathrm{p}=0.0013\right)$ and transverse $\left(R^{2}=0.9777 ; p=0.0014\right)$ measurements of the veru- montanum with rising body mass index (BMI), with statistical significance. However, in the comparison according to age, verumontanum diameters decreased (longitudinal diameter $\left(R^{2}=0.3403 ; p=0.1692\right)$ and transverse diameter $\left(\mathrm{R}^{2}=0.4221 ; \mathrm{p}=0.1142\right)$ as age increased (Figure-2).

In BPH patients who used 5-alpha-reductase inhibitors (group 1), when comparison of the verumontanum measurements and BMI revealed na increase in longitudinal diameter $\left(R^{2}=0.0285\right.$; $\mathrm{p}=0.3397)$ and a slight increase in transverse diameter $\left(\mathrm{R}^{2}=0.0005 ; \mathrm{p}=0.8986\right)$ with an increase in BMI. In this group there was a reduction in longitudinal $\left(R^{2}=0.0238 ; p=0.3833\right)$ and transverse $\left(R^{2}=0.0325\right.$; $\mathrm{p}=0.3080$ ) diameters with increasing age. In the comparson with prostate weight, there was a reduction in longitudinal $\left(\mathrm{R}^{2}=0.0237 ; \mathrm{p}=0.3852\right)$ and transverse $\left(R^{2}=0.1864 ; p=0.0108\right)$ diameters as the prostate weight increased, but only the transverse diameter was statistically significant (Figure-3).

The graphs show that the verumontanum did not increase with age in the three groups. It can also be noted that the verumontanum did not increase with increased prostate volume, suggesting that in patients with BPH there is no associated growth of the verumontanum along with the prostate.

During the anatomical analysis of the verumontanum, we observed five different morphological types, whose nomenclature we created according to their appearance (Figure-4): "Volcano" colliculus is a short colliculus with the utricle at its upper extremity;

Table 2 - Clinical characteristics of the studied groups.

\begin{tabular}{|c|c|c|c|c|}
\hline Variables & $\begin{array}{c}\text { Control }(n=7) \\
\mu \pm \partial ; m\end{array}$ & $\begin{array}{c}\text { BPH+without 5ARIs } \\
\qquad(n=52) \\
\mu \pm \partial ; m\end{array}$ & $\begin{array}{c}\text { BPH+5ARIs } \\
(n=34) \\
\mu \pm \partial ; m\end{array}$ & $P$ value \\
\hline Age (years) & $32.71 \pm 3.73 ; 32.00$ & $62.69 \pm 11.12 ; 65.00$ & $67.26 \pm 8.94 ; 68.00$ & $<0.0001^{(1)}$ \\
\hline Body mass index $\left(\mathrm{kg} / \mathrm{m}^{2}\right)$ & $27.36 \pm 3.72 ; 25.90$ & $27.11 \pm 4.19 ; 26.90$ & $26.11 \pm 4.38 ; 25.00$ & $0.4203^{(1)}$ \\
\hline Prostate weight $(\mathrm{g})$ & - & $40.85 \pm 19.80 ; 35.00$ & $59.85 \pm 23.94 ; 56.50$ & $<0.0001^{(2)}$ \\
\hline $\begin{array}{l}\text { Longitudinal diameter of the } \\
\text { verumontanum (mm) }\end{array}$ & $5.63 \pm 4.93 ; 4.26$ & $4.54 \pm 1.86 ; 4.38$ & $4.69 \pm 1.56 ; 4.52$ & $0.6990^{(1)}$ \\
\hline $\begin{array}{l}\text { Transversal diameter of the } \\
\text { verumontanum }(\mathrm{mm})\end{array}$ & $4.11 \pm 3.31 ; 2.46$ & $3.20 \pm 1.15 ; 3.03$ & $2.94 \pm 0.83 ; 3.06$ & $0.6261^{(1)}$ \\
\hline
\end{tabular}

$\mathbf{B P H}=$ Benign prostatic hyperplasia; 5 ARIs $=5$ alpha reductase inhibitors; Data were expressed as mean $(\mu) \pm$ standard deviation $(\partial)$; median $(\mathrm{m})$.

(1) Nonparametric differences were tested by Kruskal-Wallis and Dunn's posttest, $p<0.05$; (2) Nonparametric differences were tested by Mann-Whitney, $p<0.05$. 
Figure 2 - The figure shows the linear regression graphs of the control group comparing the age (years) and BMI $\left(\mathrm{kg} / \mathrm{m}^{2}\right)$ variables with the verumontanum measurements. Linear regression demonstrates that the longitudinal $\left(r^{2}=0.3403 ; p=0.1692\right)$ and transverse $\left(r^{2}=0.4221 ; p=0.1142\right)$ diameters of verumontanum decreased with age. The longitudinal $\left(r^{2}=0,9791\right.$; $\mathrm{p}=0.0013)$ and transverse $\left(\mathrm{r}^{2}=0.9777 ; \mathrm{p}=0.0014\right)$ diameters of verumontanum increased significantly with increasing BMI.
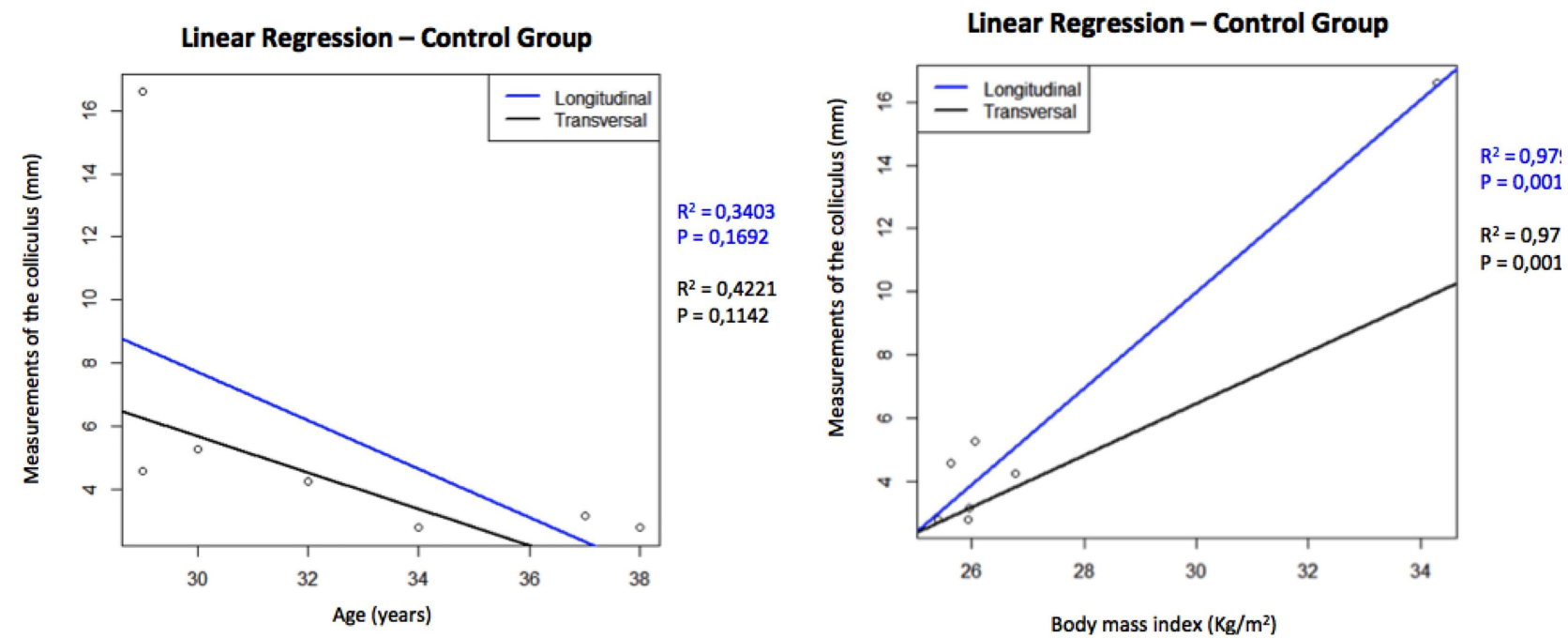

"Lighthouse" colliculus is longer colliculus with the anterior utricle at its upper extremity; "Whale Tail" colliculus is a short, flattened organ with an elongated urethral crest; "Hood" colliculus is the most elongated colliculus of all, tapered and continuous with the urethral crest; and "Castle Door" colliculus is a broad, short colliculus with enlarged prostate utricle. Group 1 presented frequency of verumontanum types as follows: 12 (35.29\%) patients with "Volcano" colliculus; 8 (23.53\%) with "Whale tail"; 7 (20.59\%) with "Lighthouse"; 4 (11.76\%) with "Hood"; and 3 (8.82\%) with "Castle Door" type. In the analysis of group 2, the frequency pattern was: 32 (61.54\%) patients with "Volcano" type colliculus; 14 (26.92\%) with "Lighthouse"; 3 (5.77\%) with "Whale Tail"; 2 (3.85\%) with "Castle Door"; and 1 (1.92\%) with "Hood" type.

In the control group, the "Volcano" colliculus was present in $4(57.14 \%)$ patients, "Lighthouse" in $2(28.57 \%)$ patients and "Whale Tail" in 1 (14.29\%) patient.

There was no statistical difference in the comparison between the three groups $(p=0.0908)$.

Of the patients who were using delayed bladder catheters, 10 (50\%) had "Volcano" colliculus, 4 (20\%) had "Whale Tail", 3 (15\%) had "Lighthouse", 2
(10\%) had "Hood", and 1 (5\%) had "Castle Door".

Among obese (BMI $\geq 30 \mathrm{~kg} / \mathrm{m}^{2}$ ), overweight (BMI 25 - $29.9 \mathrm{~kg} / \mathrm{m}^{2}$ ) and normal patients (BMI $\leq 24.9 \mathrm{~kg} / \mathrm{m}^{2}$ ), the "Volcano" verumontanum was the most frequent in all of them, presenting frequencies of 7 (46.66\%), 29 (52.72\%) and 12 (52.17\%) respectively, with statistical significance $(\mathrm{p}=0.022)$.

\section{DISCUSSION}

The 5-alpha-reductase inhibitors are known to act by inducing apoptosis of prostate epithelial cells (12), leading to a reduction in prostate size of about $18-28 \%$ and a decrease in serum prostate-specific atigen (PSA) levels of about 50\% after six to twelve months of treatment $(8,11)$. In addition, 5-alpha-reductase inhibitors improve International Prostate Symptom Score (IPSS) by $15-30 \%$ and urinary maximal flow by $1.5-2.0 \mathrm{~mL} / \mathrm{s}$ in patients with LUTS $(9,10) .5$-alpha-reductase inhibitors reduce the long-term risk ( $>$ one year) of acute urinary retention (AUR) or the need for surgery (13). In addition, finasteride can decrease bleeding during transurethral prostate resection surgery, probably due to its effects on prostate vascularization (14). 
Figure 3 - The figure shows the linear regression graphs of group 1 and group 2 comparing the variables age (years), BMI $\left(\mathrm{kg} / \mathrm{m}^{2}\right)$ and prostatic weight (grams) with the verumontanum measurements. Linear regression of group 1: A) With increasing age, the longitudinal $\left(r^{2}=0.0238 ; p=0.3833\right)$ and transverse $\left(r^{2}=0.0325 ; p=0.3080\right)$ diameters of the verumontanum decreased; $B)$ There was na increase in longitudinal $\left(r^{2}=0.0285 ; p=0.3397\right)$ and transverse $\left(r^{2}=0.0005 ; p=0.8986\right)$ diameters with increasing BMI; C) There is a reduction in longitudinal $\left(r^{2}=0.0237 ; p=0.3852\right)$ and transverse $\left(r^{2}=0.1864 ; p=0.0108\right)$ diameters with increasing prostate weight. Linear regression of group 2: D) There was na increase in longitudinal diameter $\left(r^{2}=0.0196 ; p=0.3223\right)$ and a reduction in transverse diameter $\left(r^{2}=0.0118 ; p=0.4440\right)$ with increasing age; $\left.E\right)$ There was a reduction in longitudinal diameter $\left(r^{2}=0.0006 ; p=0.8662\right)$ and an increase in transverse diameter $\left(r^{2}=0.0049 ; p=0.6235\right)$ with increasing BMI; F) There was a reduction in Iongitudinal $(r 2=0.0029 ; p=0.7063)$ and transverse $\left(r^{2}=0.0078 ; p=0.5323\right)$ diameter with increasing prostate weight.
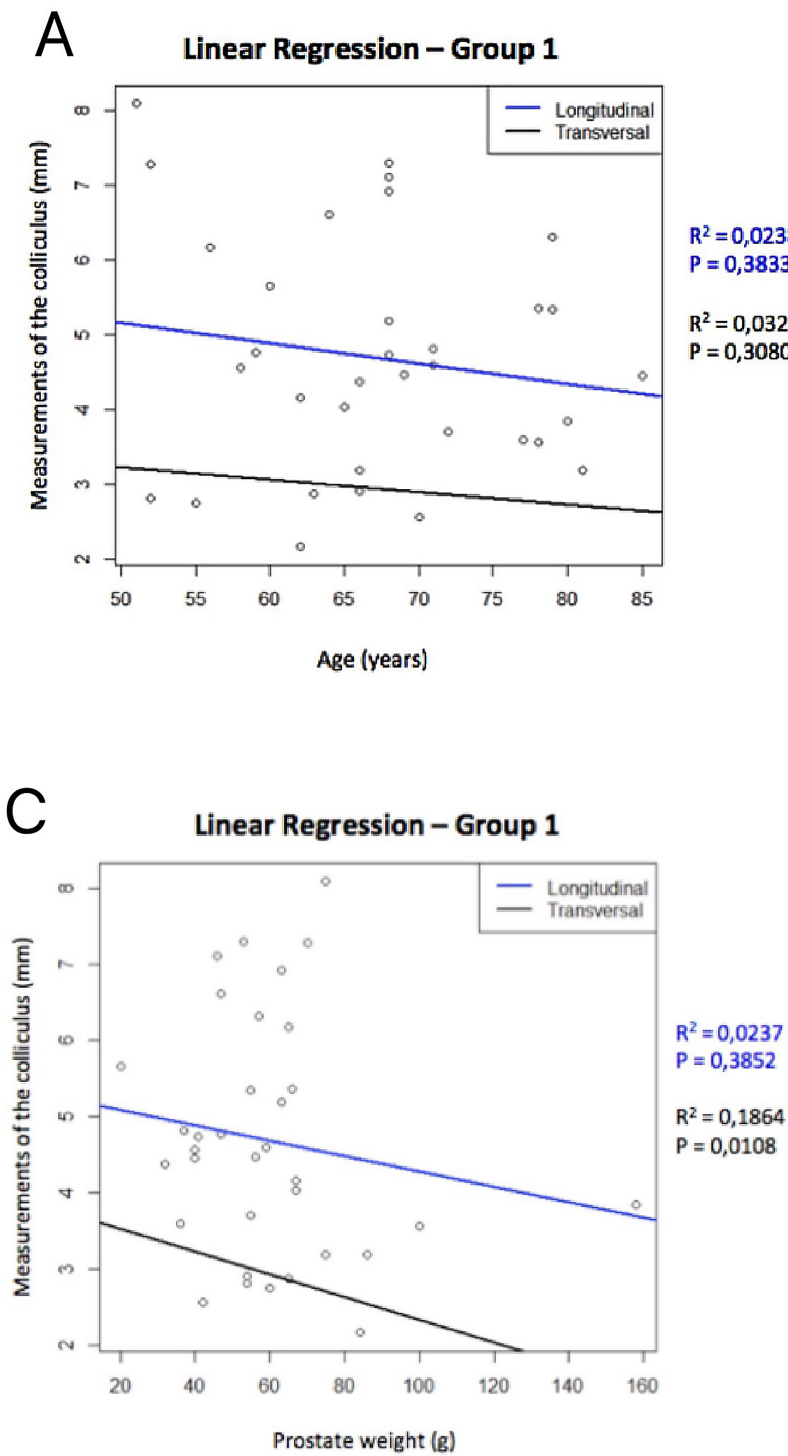
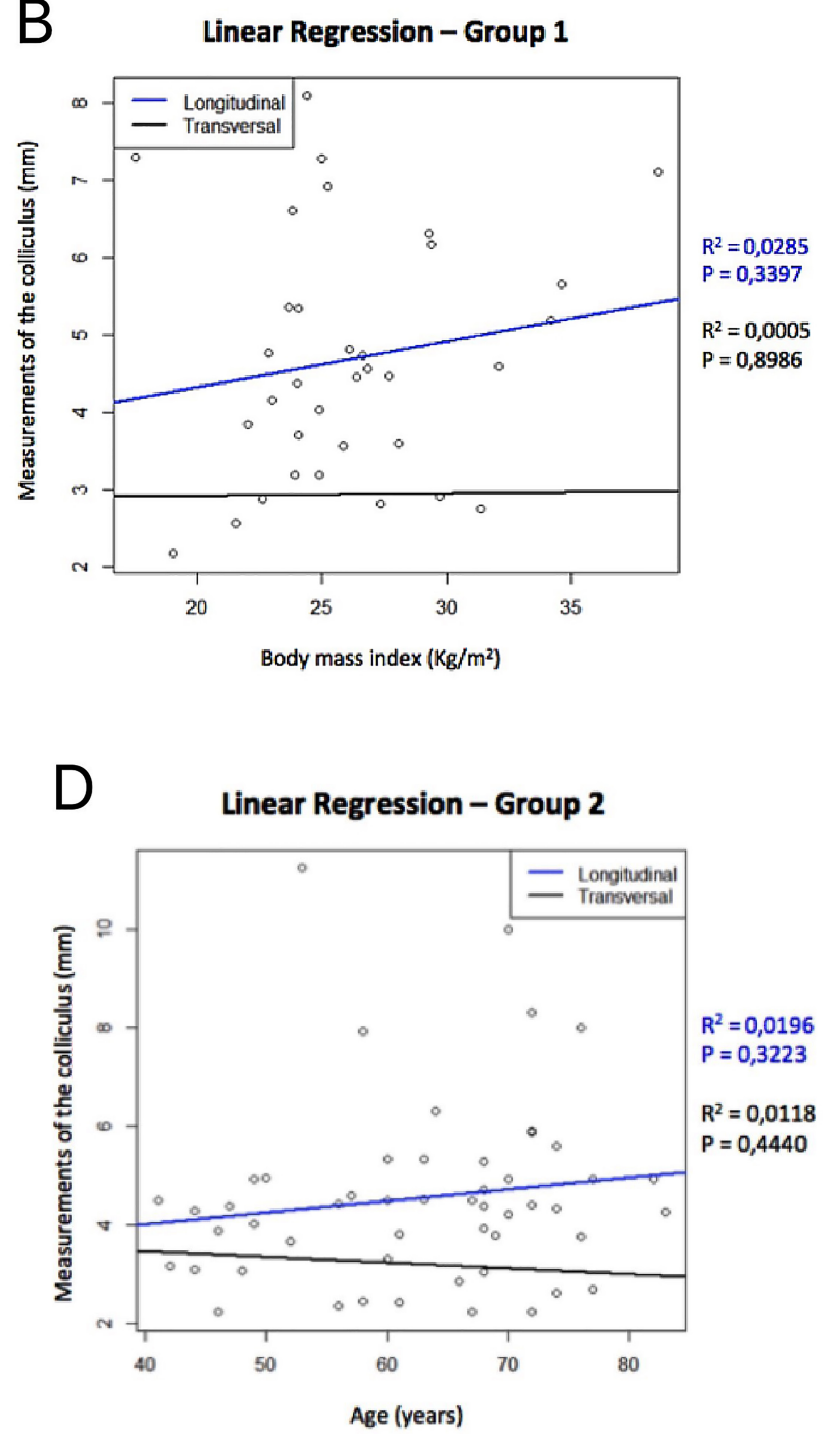


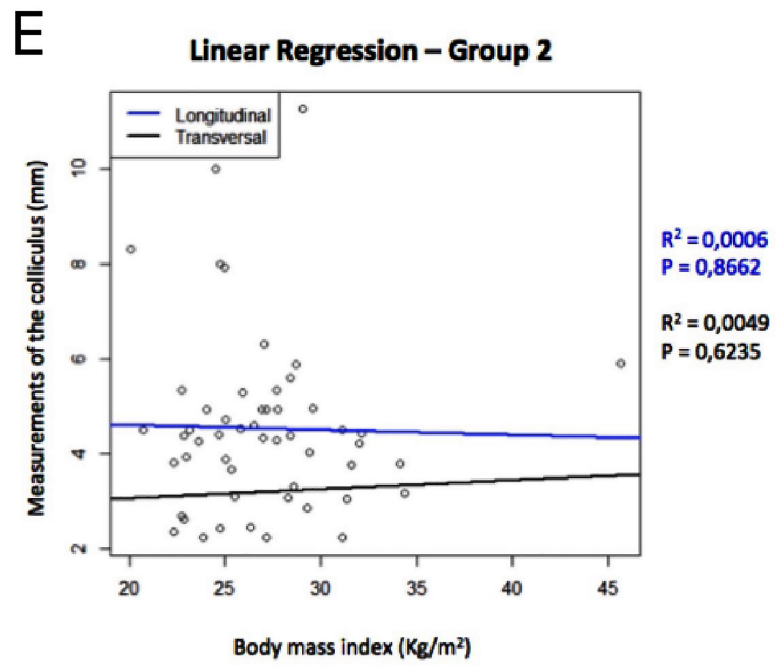

However, there is no information in the literature on whether this class of drugs alters the verumontanum size. In the present study, the mean verumontanum measurements were higher in the control group compared to the group without the drug, where the transverse diameter was larger than in the group that used the medicine. This suggests that 5-alpha-reductase inhibitors also decrease the verumontanum size.

In addition to decreased libido and erectile dysfunction, it is now known that the use of 5-alpha-reductase inhibitors can cause ejaculatory disorders and reduced semen volume $(8-10,15)$. The cause of these ejaculatory disorders is not known, but we can speculate that changes in verumontanum size could be involved. Therefore, further studies are needed to assess whether the form of verumontanum, influenced by the use of 5-ARIs in the population affected by BPH/LUTS, could modify the ejaculatory pattern of these individuals.

In our sample, in patients of group 1 and group 2, we observed a decrease in verumontanum measurements along with an increase in prostate weight, but only the decrease in verumontanum transversal measurement in the BPH group who used the drug was statistically significant.

BMI and metabolic syndrome are important in the incidence and prognosis of prostate diseases

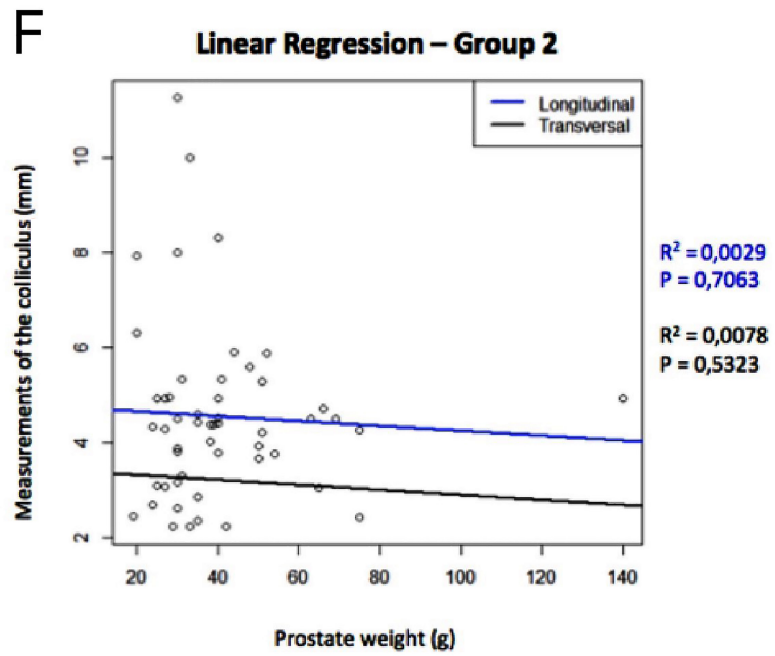

(16). There are no reports in the literature of alteration of verumontanum morphology in patients with BPH using 5-alpha-reductase inhibitors. In our study, we observed in patients who used 5-alpha-reductase inhibitors an increase in both diameters (mainly in the longitudinal diameter) with increase of BMI. In the group who did not use 5-alpha reductase inhibitors, the longitudinal diameter of the verumontanum decreased and the transverse diameter increased as the BMI increased. And in the control group, verumontanum diameters increased as BMI increased, with statistical significance.

During prostate TUR surgery, there is concern about verumontanum injury. Thus, Malalasekera and collaborators (17) performed a 3D study of the pathway of the ejaculatory ducts through the prostate to try to define a way to minimize the chance of ejaculatory duct injury during trans-urethral resection of the prostate, and he suggested preserving the prostate tissue located $7.5 \mathrm{~mm}$ on either side of the verumontanum from the midline and $10 \mathrm{~mm}$ proximal to the verumontanum. Thus, knowledge of the anatomy of the verumontanum is again important to define resection limits in the surgical treatment of BPH (17).

Another condition to be discussed would be obstructive azoospermia. This disease leads to infertility due to obstruction of the male reproductive tract, 
Figure 4 - The figure shows the 5 types of verumontanum found in the study. The left side shows the 5 types of verumontanum during the endoscopic surgery and the right has drawings of the 5 types. A) "Volcano". B) "Lighthouse". C) "Whale Tail". D) "Castle Door". E) "Hood".
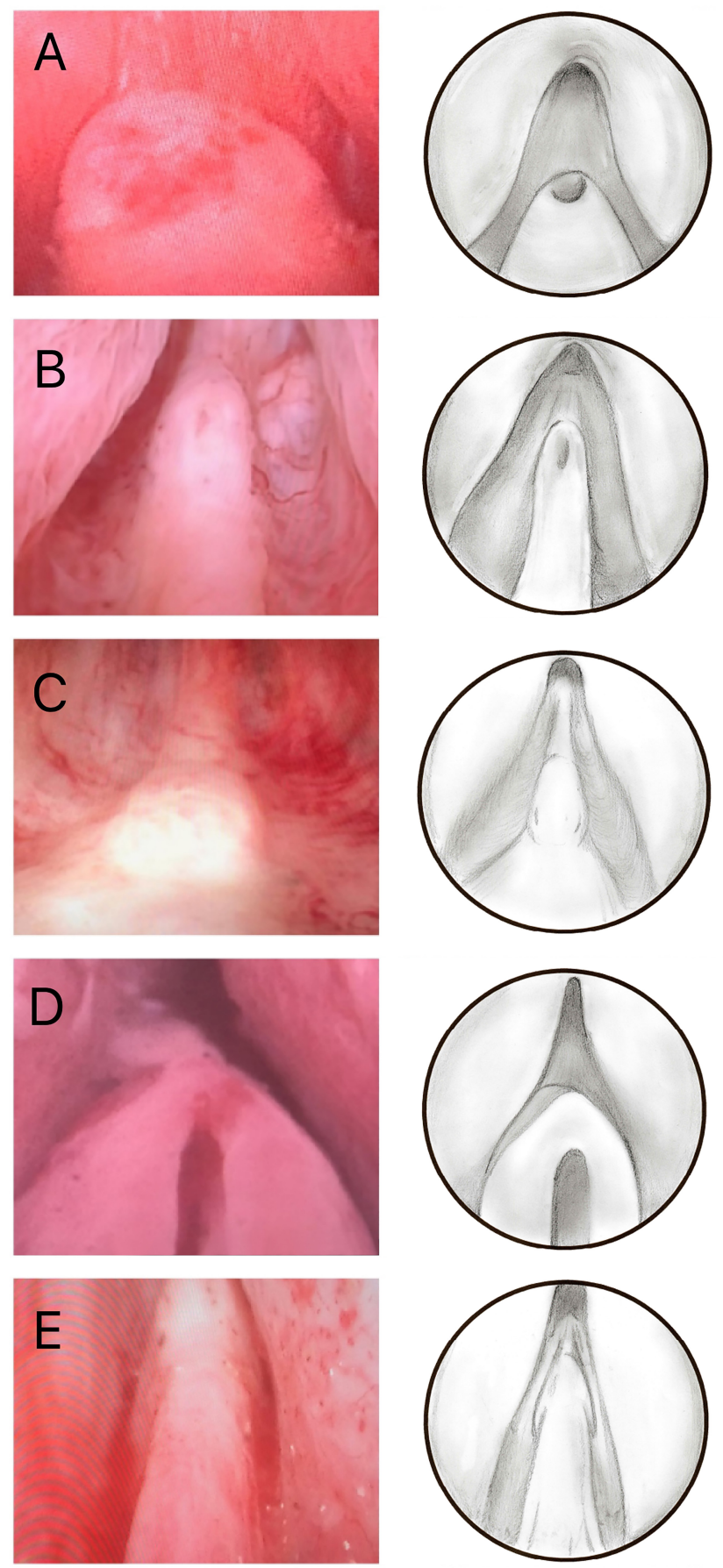

which can occur anywhere (rete testis, efferent ducts, epididymis, vas deferens and ejaculatory duct) (18). One of the tests used to diagnose this condition is seminal vasography/vesiculography, which consists of catheterization of the ejaculatory ducts through the verumontanum and contrast injection $(19,20)$. When the obstruction is located in the ejaculatory ducts, the ideal treatment is transurethral resection of the ejaculatory duct, accessed through the verumontanum (20). Thus, better knowledge of the anatomy of the verumontanum, as well as its classification, may help the endoscopic treatment of obstructive azoospermia. These facts reinforce the importance of knowledge of verumontanum anatomy.

The average diameter of verumontanum in group 3 was higher than in group 2, which was higher than in group 1, suggesting that 5-alpha-reductase inhibitors shrink the prostate as well as the verumontanum. Patients using 5-alpha-reductase inhibitors showed increased longitudinal diameter of the verumontanum with increasing BMI. In the group who did not use 5-alpha reductase inhibitors, there was a reduction in longitudinal diameter and an increase in transverse diameter of the verumontanum as the BMI increased. The verumontanum was smaller in patients with $\mathrm{BPH}$ who used and those who did not use 5-alpha-reductase inhibitors as the prostate enlarged, suggesting that BPH does not increase the size of the verumontanum. In the control group, verumontanum diameters increased with increasing BMI, suggesting that obesity may be associated with increased verumontanum size. In all groups the measures of the verumontanum decreased with advancing age, except for group 2, which presented an increase in longitudinal diameter.

A finding of great interest during this study is that all patients could be grouped into one of the five categories of our verumontanum morphological classification. From what has been shown, we believe this classification represents anatomic reality and will be useful in future studies involving the verumontanum. We propose to classify the verumontanum into five different anatomical types ("Volcano", "Lighthouse", "Whale Tail", "Hood" and "Castle Door"). We observed that the "Volcano" colliculus was the most frequent $(51.61 \%$ of all patients in the study), followed by the "Lighthouse To- 
wer" and "Whale Tail" types, with the "Castle Door" and "Hood" being less prevalent. However, we did not observe any difference between the groups, suggesting that the type of colliculus is not altered by $\mathrm{BPH}$, the use of 5-alpha-reductase inhibitors or the use of delayed bladder catheters.

The main limitations of the present study are: presence of a small sample of patients; impossibility of measuring the third diameter of the verumontanum and consequently calculating its volume, because the image analyzed by endoscopy is obtained in two dimensions; lack of standardization of a single type of 5-ARIs; our measurement method did not use more reliable measurement tools, such as a caliper, but this was the only way we found to carry out the measurements, considering that the study was with live patients.

\section{CONCLUSION}

The veromontanum measurements were smaller in patients with BPH who used and those who did not use the medicine as the prostate enlarged. In the control group, there was an increase in verumontanum diameters with an increase in BMI. We observed the presence of five morphological types of verumontanum in our sample ("Volcano", "Lighthouse", "Whale Tail", "Hood" and "Castle Door"), and the "Volcano" type was most frequent regardless of groups or BMI, suggesting that the use of 5-alpha-reductase inhibitors and obesity do not influence verumontanum morphology. Creating a new anatomical classification is always interesting. In addition, we believe this classification may help in endoscopic prostate surgery as well as future studies.

\section{ABBREVIATIONS}

$\mathrm{BPH}=$ Benign prostatic hyperplasia

LUTS $=$ Lower urinary tract symptoms

5-ARIs = 5-alpha-reductase inhibitors

$\mathrm{BMI}=$ Body mass index

DBC $=$ Delayed bladder catheter

TUR $=$ Transurethral resection

$\mathrm{OR}=$ Odds ratio

PSA $=$ Prostate-specific antigen

IPSS = International prostate symptom score

AUR $=$ Acute urinary retention

\section{ACKNOWLEDGMENTS}

The present paper was supported by FAPERJ, CAPES and CNPQ.

We would like to thank Dr. Eduardo Medina

Felici for the verumontanum illustration.

\section{CONFLICT OF INTEREST}

None declared.

\section{REFERENCES}

1. Kavoussi PK: Surgical, Radiographic, and Endoscopic Anatomy of the Male Reproductive System. In: Campbell-Walsh Urology. $11^{\mathrm{a}}$ ed. Philadelphia, Elsevier. 2016; 21: 498-515.

2. Sadler TW: Langman's Medical Embryology. 7th ed. Baltimore, Wialliams \& Wilkins. 1995.

3. Nork JJ, Yap MK, Kaplan GW. Verumontanum Cyst Associated With Lower Urinary Tract Symptoms in an Adolescent. Urology. 2016;88:192-4.

4. McNeal JE, Redwine EA, Freiha FS, Stamey TA. Zonal distribution of prostatic adenocarcinoma. Correlation with histologic pattern and direction of spread. Am J Surg Pathol. 1988;12:897-906.

5. Cifuentes Delatte L: Cirurgia Urologica Endoscopica. $2^{\mathrm{a}}$ ed Madrid, Paz Montalvo S.A. 1981.

6. Oh CS, Chung IH, Won HS, Kim JH, Nam KI. Morphologic variations of the prostatic utricle. Clin Anat. 2009;22:358-64.

7. Egan KB. The Epidemiology of Benign Prostatic Hyperplasia Associated with Lower Urinary Tract Symptoms: Prevalence and Incident Rates. Urol Clin North Am. 2016;43:289-97.

8. Andriole G, Bruchovsky N, Chung LW, Matsumoto AM, Rittmaster R, Roehrborn C, et al. Dihydrotestosterone and the prostate: the scientific rationale for 5alpha-reductase inhibitors in the treatment of benign prostatic hyperplasia. J Urol. 2004;172(4 Pt 1):1399-403.

9. McConnell JD, Roehrborn CG, Bautista OM, Andriole GL Jr, Dixon CM, Kusek JW, et al. The long-term effect of doxazosin, finasteride, and combination therapy on the clinical progression of benign prostatic hyperplasia. N Engl J Med. 2003;349:2387-98.

10. Roehrborn CG, Siami P, Barkin J, Damião R, Major-Walker $K$, Nandy I, et al. The effects of combination therapy with dutasteride and tamsulosin on clinical outcomes in men with symptomatic benign prostatic hyperplasia: 4-year results from the CombAT study. Eur Urol. 2010;57:123-31. Erratum in: Eur Urol. 2010;58:801. 
11. Naslund MJ, Miner M. A review of the clinical efficacy and safety of 5alpha-reductase inhibitors for the enlarged prostate. Clin Ther. 2007;29:17-25.

12. Rittmaster RS, Norman RW, Thomas LN, Rowden G. Evidence for atrophy and apoptosis in the prostates of men given finasteride. J Clin Endocrinol Metab. 1996;81:814-9.

13. McConnell JD, Bruskewitz R, Walsh P, Andriole G, Lieber M, Holtgrewe $H L$, et al. The effect of finasteride on the risk of acute urinary retention and the need for surgical treatment among men with benign prostatic hyperplasia. Finasteride Long-Term Efficacy and Safety Study Group. N Engl J Med. 1998;338:557-63.

14. Donohue JF, Sharma H, Abraham R, Natalwala S, Thomas DR, Foster MC. Transurethral prostate resection and bleeding: a randomized, placebo controlled trial of role of finasteride for decreasing operative blood loss. J Urol. 2002;168:2024-6.

15. Fertig RM, Gamret AC, Darwin E, Gaudi S. Sexual side effects of 5 - $\alpha$-reductase inhibitors finasteride and dutasteride: $A$ comprehensive review. Dermatol Online J. 2017;23.

16. Yin Z, Yang JR, Rao JM, Song W, Zhou KQ. Association between benign prostatic hyperplasia, body mass index and metabolic syndrome in Chinese men. Asian J Androl. 2015;17:826-30.
17. Malalasekera AP, Sivasuganthan K, Sarangan S, Thaneshan K, Weerakoon DN, Mathangasinghe Y, et al. Morphological variations of the human ejaculatory ducts in relation to the prostatic urethra. Clin Anat. 2018;31:456-61.

18. Jow WW, Steckel J, Schlegel PN, Magid MS, Goldstein M. Motile sperm in human testis biopsy specimens. J Androl. 1993:14:194-8.

19. Wosnitzer MS, Goldstein M. Obstructive azoospermia. Urol Clin North Am. 2014;41:83-95.

20. Meacham RB, Hellerstein DK, Lipshultz LI. Evaluation and treatment of ejaculatory duct obstruction in the infertile male. Fertil Steril. 1993;59:393-7.

Correspondence address: Henrique Barbosa de Menezes, MD Rua Mary Ubirajara, 110/602 - Santa Lúcia

Vitória, ES, 29056-030, Brasil

Telephone.: +55 27 98177-6300

E-mail: henriquebmenezes@hotmail.com 